Zugang zum Staat 


\section{Demokratische Gleichheit als gerechte Staatlichkeit Eine antidiskriminierungsrechtliche Perspektive auf den Zugang zu Staatsämtern und Parität}

Dr. Cara Röhner

Abstract: Recht wird in staatlichen Institutionen erschaffen, durchgesetzt und gesprochen. Personell sind staatliche Institutionen jedoch recht homogen besetzt und repräsentieren nicht die pluralistische Gesellschaft. Die Ungleichheitsforschung hat gezeigt, dass die zahlenmäßige Unterrepräsentation von dominierten Gruppen auf strukturelle Zugangsbarrieren hinweist. In dem Beitrag wird daher argumentiert, dass demokratische Gleichheit über die formale Rechtsgleichheit hinaus eine antidiskriminierungsrechtliche Dimension umfassen muss, um den diskriminierungsfreien Zugang zu Staatsämtern - und in diesem Sinne gerechte Staatlichkeit - zu gewährleisten. Dafür wird zunächst eine demokratietheoretische und antidiskriminierungsrechtliche Begründung für politische Maßnahmen, die einen gleichen Zugang zum Staat gewährleisten sollen, entworfen. Anschließend werden die verfassungsrechtlichen Entscheidungen zu den Paritätsgesetzen diskutiert und eingeordnet.

\section{Einleitung: Strukturelle Ungleichheiten im Staat}

Recht wird in staatlichen Institutionen erschaffen, durchgesetzt und gesprochen. Personell sind staatliche Institutionen jedoch recht homogen besetzt und repräsentieren nicht die pluralistische Gesellschaft. Dieses Ungleichgewicht zeigt sich insbesondere bei den legislativen, exekutiven und judikativen Spitzenämtern: Je machtvoller die Position ist, desto stärker dominiert die hegemoniale Subjektposition. Die hegemoniale Subjektposition wird im materialen Gleichheitsdiskurs als die gesellschaftlich dominierende Gruppe beschrieben. Die Unterscheidung zwischen einer dominierenden und dominierten Gruppe verweist auf gesellschaftliche Ungleichheitsverhältnisse, die dazu führen, dass materielle und immaterielle Ressourcen - und eben auch staatliche Ämter - in einer Gesellschaft 
ungleich verteilt sind. ${ }^{1}$ Die gesellschaftlich dominante Position bezeichnet das „Normale“ und das „Allgemeine“, die dominierte Position die „Abweichung" und das „Besondere“. Während die »normale« Position nicht benannt werden muss, ist die gesellschaftlich dominierte Ausprägung als "das Andere « aktiv markiert. ${ }^{2}$ Ein materiales Gleichheitsverständnis legt also ein asymmetrisches Gesellschaftsbild zugrunde und schreibt dem Staat die Aufgabe zu, tatsächliche Gleichheit durch politische Maßnahmen zu realisieren.

Die Unterrepräsentation von nicht-hegemonialen Subjektivitäten bzw. dominierten Gruppen im Staat zeigt sich exemplarisch an der Geschlechterungleichheit. In den Parlamenten liegt der Anteil der Mandatsträgerinnen regelmäßig zwischen 25 und 35 Prozent, in den kommunalen Vertretungen zum Teil deutlich darunter. ${ }^{3}$ Auch kann nicht von einer langsamen, aber kontinuierlichen Fortschrittsgeschichte ausgegangen werden. Nach den letzten Bundestags- und Landtagswahlen lässt sich ein deutlicher Rückgang des Frauenanteils beobachten. Im Deutschen Bundestag beträgt er nach der letzten Wahl nicht mehr 36 Prozent, sondern nur noch 30 Prozent. In den Landtagen von Brandenburg, Sachsen, Thüringen und Rheinland-Pfalz sind die Frauenanteile ebenfalls um 2 bis 11 Prozentpunkte gesunken. ${ }^{4}$ Dies ist insbesondere auf die Wahlerfolge von Parteien zurückzuführen, die parteiintern über keine effektive Verpflichtung zu einer geschlechtergerechten Nominierung verfügen.

Die Unterrepräsentation zeigt sich nicht nur in den Parlamenten, sondern auch in den anderen zwei Staatsgewalten. Auch hier gilt: Je höher die Hierarchieebene, desto homogener die Besetzung der staatlichen Ämter. Nach der Regierungsbildung im Frühjahr 2018 wurden z.B. auf der

1 Sacksofsky, Das Grundrecht auf Gleichberechtigung. Eine rechtsdogmatische Untersuchung zu Artikel 3 Absatz 2 des Grundgesetzes, 2. Aufl., 1996; Baer, Würde oder Gleichheit? Zur angemessenen grundrechtlichen Konzeption von Recht gegen Diskriminierung am Beispiel sexueller Belästigung am Arbeitsplatz in der Bundesrepublik Deutschland und den USA, 1995.

2 Mangold, Demokratische Inklusion durch Recht. Antidiskriminierungsrecht als Ermöglichungsbedingung der demokratischen Begegnung von Freien und Gleichen, 2020, S. 5 ff., pointiert: »Menschen haben also kein Geschlecht, solange sie männlich sind «(S. 6). Siehe auch die Beiträge in Klinger et al., Achsen der Ungleichheit. Zum Verhältnis von Klasse, Geschlecht und Ethnizität, 2007.

3 Aus der Politikwissenschaft zur kommunalen Ebene z.B. Holtkamp/Wiechmann, Genderranking deutscher Großstädte 2013, 2013; Kletzing/Lukoschat, Engagiert vor Ort - Wege und Erfahrungen von Kommunalpolitikerinnen, 2. Aufl. 2011.

4 Aufgrund der Wahlerfolge von Bündnis90/Die Grünen ist der Frauenanteil allein im Landtag von Baden-Württemberg von 24,5 auf 29,2 Prozent angestiegen. 
Leitungsebene der Bundesministerien nur 4 von 29 verbeamteten Staatssekretärsämter an Frauen vergeben. ${ }^{5}$ Für die Bundesverwaltung insgesamt lag der Frauenanteil im Jahr 2019 zwar bei 54 Prozent der Gesamtbeschäftigten, aber nur bei 36 Prozent bei den Leitungsfunktionen. ${ }^{6}$ Diese Ämterverteilung lässt sich auch in der Gerichtsbarkeit beobachten. Während Richterinnen in der Justiz bundesweit inzwischen mit über 45 Prozent vertreten sind, ${ }^{7}$ liegt ihr Anteil an den höheren Richterämtern deutlich darunter. Am Bundesgerichtshof beträgt der Frauenanteil z.B. ein Drittel. ${ }^{8}$ Eine Ausnahme stellt die Ämterverteilung am Bundesverfassungsgericht dar. Gegenwärtig sind neun der sechzehn Ämter weiblich besetzt. Dies ist durch die frauenorientierte Nominierungspolitik von Bündnis90/Die Grünen und der SPD zu erklären. Dennoch kann auch hier beobachtet werden, dass das höchste Amt - das des Bundesverfassungsgerichtspräsidentens - erst kürzlich erneut männlich besetzt wurde.

Die Ungleichheitsforschung hat gezeigt, dass die zahlenmäßige Unterrepräsentation von dominierten Gruppen auf strukturelle Zugangsbarrieren hinweist. ${ }^{9}$ Für das Geschlechterverhältnis gelten geschlechterstereotype Deutungsmuster und Sozialisationsprozesse, die ungleiche Verteilung von Sorge- und Pflegeverantwortung sowie das Fortwirken des historischen Ausschlusses aus der Öffentlichkeit als wesentliche strukturelle Ursachen für die fortbestehende Unterrepräsentation von Frauen in staatlichen Ämtern und Führungspositionen. Die zahlenmäßige Unterrepräsentation kann danach als Ausdruck der ungleichen gesellschaftlichen Machtverhältnisse, die sich in staatlichen Institutionen manifestieren, verstanden werden.

5 Biermann, Gleichstellungsbeauftragte beschweren sich bei Merkel, Die Zeit online v. 25.04.2018, https:/www.zeit.de/politik/deutschland/2018-04/gleichstellung-merk el-staatssekretaer-gleichstellungsbeauftragte (Stand: 18.04.2021).

6 Statistisches Bundesamt, Gleichstellungsindex 2020. Gleichstellung von Frauen und Männern in den obersten Bundesbehörden, 2021, 6 ff.; Entwurf eines Gesetzes zur Ergänzung und Änderung der Regelungen für die gleichberechtigte Teilhabe von Frauen an Führungspositionen in der Privatwirtschaft und im öffentlichen Dienst, BT-Drucksache 19/26689, S. 2.

7 Bundesamt für Justiz, Zahl der Richter, Richterinnen, Staatsanwälte, Staatsanwältinnen und Vertreter, Vertreterinnen des öffentlichen Interesses in der Rechtspflege der Bundesrepublik Deutschland am 31. Dezember 2018.

8 Deutscher Juristinnenbund, Offener Brief an den Richterwahlausschuss: Faire Berücksichtigung von Frauen bei den Bundesrichterwahlen am 2. Juli 2020, Pressemitteilung v. 23.06.2020 zur Initiative „Frauen in Roten Roben“.

9 Sacksofsky, ZESAR 2004, 208. 
Bei Einstellungen, Ernennungen, Beförderungen und Nominierungen wirkt zudem das Ähnlichkeitsprinzip: Menschen nehmen solche, die ihnen ähnlich sind, als erfolgreich wahr und wählen diese aus. ${ }^{10}$ In politischen Parteien, staatlichen Behörden und Gerichten mit einem relativ homogenen Führungspersonal stellt dies eine strukturelle Hürde für den Zugang von Angehörigen dominierter Gruppen dar. Die australische Verfassungsrichterin Helen Irving stellt daher für die Besetzung von Ämtern im Staat fest: „Wo die Übernahme eines Amtes von der Wahl oder den Präferenzen derjenigen abhängt, die bereits an der Macht sind, wird die Kultur dazu neigen, das zu perpetuieren, was sich bereits etabliert hat." ${ }^{11}$

Diese exkludierende Dynamik zeigt sich nicht nur für das Geschlechterverhältnis, sondern auch für andere Achsen der Ungleichheit wie etwa der Unterrepräsentation von Menschen mit Migrationsgeschichte in den staatlichen Institutionen. ${ }^{12}$ In Berlin wurde zu Beginn des Jahres 2021 erstmalig in Deutschland die Einführung einer Diversitätsquote diskutiert. Der Anteil von Menschen mit Migrationsgeschichte, der in der Stadtbevölkerung bei etwa 35 Prozent liegt, wird in der Berliner Verwaltung auf 12 Prozent geschätzt. Die Berliner Sozialsenatorin schlug daher vor, eine Quote entsprechend des Bevölkerungsanteils für die Berliner Verwaltung einzuführen, um Chancengleichheit für Menschen mit Migrationsgeschichte herzustellen und struktureller Diskriminierung entgegenzuwirken. ${ }^{13}$ Die Diversitätsquote war in der rot-rot-grünen Regierungskoalition jedoch nicht konsensfähig, weshalb sich diese auf - weniger effektive freiwillige Zielvorgaben und Förderpläne geeinigt hat.

10 Für die deutsche Wirtschaft: AllBright-Stiftung, Der ewige Thomas-Kreislauf?, 2017. Für die deutsche Exekutive: Biermann et al., Die Hans-Bremse, Die Zeit v. 08.10.2018. Aus sozialwissenschaftlicher Sicht der Netzwerkforschung z.B. Sagebiel, Netzwerke: soziales Kapital und Macht(unterschiede) von Frauen und Männern, in: Kortendiek et al. (Hrsg.), Handbuch Interdisziplinäre Geschlechterforschung, 2019.

11 Irving, Gender and the Constitution. Equity and Agency in Comparative Constitutional Design, 2008, S. 113, O.i.Engl., eigene Übersetzung.

12 Wisser, Eignung und Repräsentation im Polizeidienst, i.V.; Ellebrecht/Wisser, Spieglein, Spieglein an der Wand, wer ist Polizei in diesem Land. Eignung für den Polizeidienst in Deutschland und die Frage der Repräsentation, in: Groß/ Schmidt (Hrsg.), Empirische Polizeiforschung XXIII. Polizei und Migration, 2020, S. 292-296.

13 Senatsverwaltung für Integration, Arbeit und Soziales, Einigung beim Gesetzesentwurf zum Partizipations- und Migrationsgesetz - Menschen mit Migrationshintergrund werden gezielt gefördert, Pressemitteilung v. 05.02.2021. 
Quotenregelungen, wie die Diversitätsquote und Paritätsgesetze für die Parlamente, gelten als eine Antwort auf die anhaltende Unterrepräsentation vielfältiger Subjektivitäten im Staat. Seit dem Thüringen und Brandenburg die ersten deutschen Paritätsgesetze erlassen haben, wird über deren Verfassungsmäßigkeit kontrovers diskutiert. Da die Ungleichheit im Staat weder auf die Parlamente noch auf das Geschlechterverhältnis beschränkt ist, kann diese Debatte breiter als Kontroverse um demokratische Gleichheit, Gleichheit im Staat und gerechte Staatlichkeit verstanden werden.

Im Folgenden wird argumentiert, dass demokratische Gleichheit über die formale Rechtsgleichheit hinaus eine antidiskriminierungsrechtliche Dimension umfassen muss, ${ }^{14}$ um den diskriminierungsfreien Zugang zu Staatsämtern - und in diesem Sinne gerechte Staatlichkeit - zu gewährleisten. ${ }^{15}$ Dies wird am Beispiel der Geschlechterungleichheit in den Parlamenten in zwei Schritten entfaltet: Zunächst wird eine demokratietheoretische und antidiskriminierungsrechtliche Begründung für politische Maßnahmen, die einen gleichen Zugang zum Staat gewährleisten, entworfen (II.), um dann die verfassungsrechtlichen Entscheidungen zu Paritätsgesetzen zu diskutieren (III.). Der Beitrag schließt mit einem Ausblick (IV.).

\section{Gerechte Staatlichkeit: Demokratische Gleichheit der Staatsbürger:innen}

Der demokratische Staat gründet in der Egalität der Staatsbürger:innen. Diese Gleichheit wird für den Zugang zu Staatsämtern streng formal verstanden. ${ }^{16} \mathrm{Im}$ Wahlrecht drückt sich die formale Gleichheit beispielsweise in der numerischen Stimmgleichheit und dem strikten Differenzierungsverbot aus. ${ }^{17}$ Im Diskurs um die Einführung von Quoten oder Paritätsgesetzen wird die formale Gleichheitsperspektive als Begründung genannt, um diese Maßnahmen als unvereinbar mit der repräsentativen Demokratie

14 Röhner, Der Staat 59 (2020), 414 (437 ff.).

15 Röhner, djbZ 22 (2019), 125.

16 BVerfG, Urt. v. 23.01.1957 - 2 BvE 2/56, BVerfGE 6, 84 (91); Wild, Die Gleichheit der Wahl. Dogmengeschichtliche und systematische Darstellung, 2003; Starck, $\$ 33$ Grundrechtliche und demokratische Freiheitsidee, in: Isensee/Kirchhof (Hrsg.), HDStR, Bd. III: Demokratie - Bundesorgane, 3. Aufl. 2005; ausführlich dazu Röhner, Ungleichheit und Verfassung. Vorschlag für eine relationale Rechtsanalyse, 2019, S. $276 \mathrm{ff}$.

17 Z.B. BVerfG, Urt. v. 03.07.2008 - 2 BvC 1, 7/07, BVerfGE 121, 266 (295); BVerfG, Urt. v. 25.07.2012 - 2 BvF 3/11, 2 BvR 2670/11, 2 BvE 9/11, BVerfGE 131, 316 (334). 
und der Wahlrechtsgleichheit der Staatsbürger:innen zu bewerten. ${ }^{18}$ In dieser Argumentation wird also die gesetzliche Maßnahmen, die auf die Herstellung von Chancengleichheit und die Bekämpfung von struktureller Diskriminierung gerichtet ist, selbst zur Demokratie- und Gleichheitsgefährdung. Die strukturelle Diskriminierung dominierter Gruppen beim Zugang zu staatlichen Ämtern wird demgegenüber weder als Problem repräsentativer Demokratie noch als Problem staatsbürgerlicher Gleichheit gesehen. Die Egalität der Staatsbürger:innen wird in dieser Lesart individualisiert und umfasst nicht den Schutz vor strukturellen Zugangsbarrieren.

Die bisher vorherrschende individualisierende, formale Perspektive auf staatliche Institutionen muss für die Adressierung von struktureller Ungleichheit nicht aufgegeben, sondern - so das folgende Argument - nur durch eine relationale, d.h. materiale-kontextuelle, Perspektive ergänzt werden. ${ }^{19}$ Durch die Ergänzung kann der Schutzgehalt der demokratischen Gleichheit fallgruppenorientiert ausdifferenziert werden. ${ }^{20}$ Die strukturelle Diskriminierung beim Zugang zu staatlichen Ämtern stellt dann eine Fallkonstellation und auch ein Problem der Egalität der Staatsbürger:innen dar. Eine solche materiale Perspektive auf die staatsbürgerliche Gleichheit lässt sich demokratietheoretisch (1.) und antidiskriminierungsrechtlich (2.) begründen.

\section{Historischer Ausschluss aus dem Staat und die Anerkennung als Gleiche}

Die historischen Erfahrungen des Ausschlusses aus dem Staat zeigen, dass Ungleichheit in die Demokratiegeschichte eingeschrieben ist. ${ }^{21}$ So waren z.B. Frauen in Deutschland bis zur Weimarer Republik vom Wahlrecht ausgeschlossen. Auch nach dem Faschismus waren Frauen in der Bonner Republik durch das Leitbild der westdeutschen Hausfrau nicht gleichberechtigt in der Öffentlichkeit inkludiert. Der Frauenanteil im Deutschen Bundestag blieb dementsprechend bis in die 1980er Jahre bei unter 10

18 Zum Diskurs Rabe in diesem Band.

19 Für eine solche relationale Perspektive siehe Röhner (Fn. 16).

20 Zur historischen Kontextualisierung und Fallgruppenbildung siehe Weber (S. 64) in diesem Band mit Verweis auf Lepsius.

21 Ausführlich m.w.N. Gerhard, Gleichheit ohne Angleichung. Frauen im Recht, 1990; Maihofer, Geschlecht als Existenzweise. Macht, Moral, Recht und Geschlechterdifferenz, 1995; Rubio-Marín, International Journal of Constitutional Law 2014, 4; Röhner (Fn. 16), S. 19 ff., 267 ff. 
Prozent und damit auf dem gleichen Niveau wie bei der ersten demokratischen Wahl im Jahr 1919. Erst durch die Wahlerfolge der Grünen und die Selbstverpflichtung einiger Parteien auf eine geschlechtergerechte Nominierung kam es zu einem Anstieg des Frauenanteils auf etwa ein Drittel.

Die Verfassungsrechtlerinnen Blanca Rodríguez-Ruiz und Ruth RubioMarín argumentieren, dass der historische Ausschluss aus der Demokratie in der geschlechtlich codierten Trennung von privater und staatlicher Sphäre fortwirke. ${ }^{22}$ Dieses Strukturmoment der repräsentativen Demokratie manifestiere sich in der anhaltenden Unterrepräsentation von Frauen in den Parlamenten. Die repräsentative Demokratie sei daher durch exkludierende Strukturen geprägt und müsse erst in eine inklusive Institution transformiert werden. Politische Maßnahmen wie Paritätsgesetze dienten dazu, eine gleichberechtigte Anwesenheit der Geschlechter in den Parlamenten zu gewährleisten und demokratische Gleichheit in der repräsentativen Demokratie herzustellen (parity democracy model). ${ }^{23}$

Mit einer gleichberechtigten Anwesenheit werden nicht nur historische Ausschlüsse überwunden, sondern auch vormals Ungleiche als politisch Gleiche anerkannt - so die zentrale Einsicht der politischen Theoretikerin Anne Phillips. ${ }^{24}$ In einer gleichen Anwesenheit komme die Anerkennung als politisch Gleiche zum Ausdruck. Daher müsse in der repräsentativen Demokratie durch politische Maßnahmen die Inklusion historisch Ausgeschlossener gewährleistet werden. Phillips begründet damit Geschlechterquoten und die Neuziehung von Wahlkreisgrenzen zugunsten von Minderheiten in den USA. ${ }^{25}$

Phillips ist in der Debatte um die Repräsentation von „Geschlechteroder Gruppeninteressen“ besonders gewinnbringend, weil sie im Kern nicht für Repräsentation, sondern für ein demokratisches Antidiskriminierungsrecht argumentiert. Sie geht in ihrer Konzeption nicht von authentischen weiblichen Interessen oder einem repräsentierbaren Willen der "Gruppe der Frauen“" aus. Es findet daher keine Essentialisierung weiblicher Identitäten statt. Stattdessen legt Phillips einen potentiell geteilten Erfahrungshorizont zugrunde, nach der die gesellschaftlichen Ungleichheitsverhältnisse dazu führen, dass bestimmte Erfahrungen überwiegend von einer Gruppe gemacht werden und somit vergeschlechtlicht sind. Eine

22 Rodríguez-Ruiz/Rubio-Marín, On Parity, Interdependence, and Women's Democracy, in: Baines/Barak-Erez/Kahana (Hrsg.), Feminist Constitutionalism. Global Perspectives, 2012, S. 188-203 (199 ff.).

23 Ebenda.

24 Phillips, The Politics of Presence, 2004, Kap. 2.

25 Ebenda, Kap. 3 und 4. 
gleichberechtigte Präsenz von Frauen in den Parlamenten könnte daher zu einer Pluralisierung der Perspektiven und der verstärkten Einspeisung von weiblichen Erfahrungswissen in die parlamentarischen Prozesse führen. ${ }^{26}$ In diesem Sinne kann Phillips Konzeption als ein Beitrag zu einem pluralistischen Demokratieverständnis gelesen werden. ${ }^{27}$

Phillips betont jedoch - und das ist zentral für die Debatte - das Fehlen einer Garantie dafür, dass eine verstärkte Präsenz von Frauen auch zu einer Politik für Frauen führt. Darauf kommt es Phillips nicht an, weil - vor dem Hintergrund des historischen Ausschlusses und seinem Fortwirken in der Gegenwart - bereits die gleichberechtigte Präsenz ein demokratisches Prinzip darstellt.

Phillips geht es also nicht um die Repräsentation von als spezifisch „weiblich“ verstandenen Inhalten und Identitäten, sondern um den gleichen Zugang von Subjekten, die historisch von der Demokratie ausgeschlossen waren und auch heute noch strukturell benachteiligt sind. Es geht also im Kern um die Gewährleistung eines diskriminierungsfreien Zugangs zur demokratischen Staatsgewalt.

Demokratische Gleichheit kann mit den dargestellten Autorinnen also als demokratisches Antidiskriminierungsrecht konzipiert werden, das auf die Anerkennung als Gleiche und die Gewährleistung eines diskriminierungsfreien Zugangs zum Staat gerichtet ist, mit dem Ziel, den historischen Ausschluss zu überwinden und eine gerechte Staatlichkeit zu schaffen.

\section{Demokratische Gleichheit als Gewährleistung eines diskriminierungsfreien Zugangs}

Antidiskriminierungsrecht soll gewährleisten, dass Menschen weder unmittelbar noch mittelbar Erfahrungen von Benachteiligung machen, weil sie einer bestimmten sozialen Gruppe angehören oder zugehörig erscheinen. ${ }^{28}$ Es soll auch vor struktureller Diskriminierung schützen und umfasst daher nicht nur Diskriminierungsverbote, sondern erlaubt der demokratischen Politik darüber hinaus, fördernde Maßnahmen zu erlassen. Die-

26 Wapler, JöR 67 (2019), 427-455; dies., Die Crux mit der Quote - Paritätsgesetze und demokratische Repräsentation, 2019; Young, Inclusion and Democracy, 2000, Kap. 4.

27 Ausführlich zur pluralistischen Demokratie Röhner, Der Staat 59 (2020), 421 (425 ff.).

28 Grundlegend Mangold (Fn. 2), S. 4 ff., 347 ff. 
se gleichheitschützende und gleichheitermöglichende Funktionen von Antidiskriminierungsrecht betont Anna Katharina Mangold. Sie zeigt auf, dass Antidiskriminierungsrecht eine Ermöglichungsbedingung der demokratischen - d.h. diskriminierungsfreien - Begegnung von Freien und Gleichen ist. Auf das deliberative Demokratieverständnis von Jürgen Habermas aufbauend argumentiert Mangold, dass „Antidiskriminierungsrecht die Voraussetzungen von öffentlicher Deliberation in der Demokratie absichert, indem es die Möglichkeit gleichrangiger demokratischer Begegnung von Bürger"innen auf Augenhöhe schafft. "29 Antidiskriminierungsrecht habe daher eine Ermöglichungsfunktion: Es eröffne die Chance auf gleichberechtigte Teilnahme am öffentlichen Diskurs auch für jene, die aufgrund intentionaler oder struktureller Exklusion mit ihren Positionen und Argumenten bislang nicht gehört worden seien. Zur gleichberechtigten Teilnahme am öffentlichen Diskurs hat Jürgen Habermas bereits den Zusammenhang zwischen Teilhabe und Selbstbestimmung im demokratischen Rechtsstaat unterstrichen und formuliert, dass es einer gleichberechtigten Teilnahme an der Praxis der staatsbürgerlichen Selbstbestimmung bedarf, "weil nur die Betroffenen selbst die jeweils >relevanten Hinsichten von Gleichheit und Ungleichheit klären können. «30

Während Mangold die demokratische Legitimation von privatrechtlichem Diskriminierungsschutz begründet, besitzt ihr Argument auch für den demokratischen Staat Gültigkeit. ${ }^{31}$ Auch im staatlichen Bereich bedarf es eines Antidiskriminierungsschutzes, um eine gleichberechtigte Teilnahme an demokratischen-rechtsstaatlichen Prozessen zu sichern. ${ }^{32}$ Menschen, die von Diskriminierung betroffen sind, sind auf Diskriminierungsschutz angewiesen, um gleichberechtigte Zugangschancen zu staatlichen Ämtern zu erhalten und an den demokratisch-rechtsstaatlichen Auseinandersetzungen - sei es in der Legislative, Exekutive oder Judikative - teilhaben zu können.

Diese Perspektive ist nicht auf die Geschlechterungleichheit beschränkt. Antidiskriminierungsrecht umfasst den Schutz aufgrund verschiedener sozialer Zugehörigkeiten. Die Diskussion um die Berliner Diversitätsquote hat bereits gezeigt, dass in einer Migrationsgesellschaft die Teilhabe von Menschen mit Migrationsgeschichte verstärkt Zugangsfragen aufwirft.

29 Ebenda, S. 397.

30 Habermas, Faktizität und Geltung. Beiträge zu einer Diskurstheorie des Rechts und des demokratischen Rechtsstaats, 1992, S. 506.

31 Röhner, Der Staat 59 (2020), 421 (437 ff.).

32 Zur staatswillenbildenden Funktion der Parteien und Paritätsregelungen Völzmann, DVBl 2021, 496. 
Auch hier dürften u.a. rassifizierende Stereotype und Deutungsmuster über Fähigkeiten und Kompetenzen dazu führen, dass Personen, denen eine nicht-deutsche Identität zugeschrieben wird, faktisch nicht die gleichen Chancen haben, eingestellt, ernannt, befördert oder nominiert zu werden.

Verfassungsrechtlich kann ein antidiskriminierungsrechtliches Verständnis demokratischer Gleichheit in den Gleichheitsrechten und dem Demokratieprinzip verankert werden. Ausgangspunkt dafür ist ein materiales Gleichheitsverständnis, wie es die Literatur und das Bundesverfassungsgericht für die Geschlechterungleichheit entwickelt haben. ${ }^{33}$ Ein materiales Gleichheitsverständnis bezieht sich auf die soziale Wirklichkeit und ist auf die Durchsetzung von tatsächlicher Gleichheit gerichtet. Die Ungleichheitsforschung hat zu der Erkenntnis geführt, dass formale Rechtsgleichheit für die Erreichung von de-facto Gleichheit nicht ausreichend ist, weil insbesondere neutrale Regelungen im Kontext einer durch Ungleichheit geprägten Gesellschaft zu faktischen Benachteiligungen führen (mittelbare Diskriminierung) ${ }^{34}$ Die gesellschaftlichen Ungleichheitsverhältnisse können sich ohne ausgleichende Regelungen in die staatlichen Institutionen einschreiben und auf diese Weise die demokratische Gleichheit von Angehörigen dominierter Gruppen beeinträchtigen. Diese strukturelle Benachteiligung darf temporär - bis zum Erreichen von de-facto Gleichheit - durch proaktives Gleichstellungsrecht ausgeglichen werden. ${ }^{35}$

Für die Geschlechterungleichheit ergibt sich aufgrund von Art. 3 Abs. 2 S. 2 GG eine staatliche Verpflichtung, die Gleichberechtigung in allen staatlichen Institutionen - in der Legislative, in der Exekutive und in der Judikative - zu verwirklichen. Darüber hinaus darf aus einer materialen Perspektive die demokratische Politik auch Fördermaßnahmen zugunsten Angehöriger anderer dominierter Gruppen, die in Art. 3 Abs. 3 GG genannt sind, erlassen, um faktischen Nachteilen und Zugangsbarrieren entgegenzuwirken. ${ }^{36}$

33 Sacksofsky (Fn. 1); Baer (Fn. 1); Mangold (Fn. 2), S. 181 ff.; Röhner, djbZ 22 (2019), 161 ff.; statt vieler: BVerfG, Urt. v. 28.01.1992 - 1 BvR 1025/82, 1 BvL 16/83 und 10/91, BVerfGE 85, 191, Nachtarbeitsverbot.

34 Z.B. BVerfG, Beschl. v. 05.04.2005 - 1 BvR 774/02, BVerfGE 113, 1; BVerfG, Beschl. v. 18.06.2008 - 2 BvL 6/07, BVerfGE 121, 241.

35 Völkerrechtlich ergibt sich dies auch aus Art. 4 Abs. 1 CEDAW.

36 Mangold (Fn. 2), S. 290 ff., 300 f.; Baer/Markard, in: Mangoldt/Klein/Starck (Hrsg.), Kommentar zum Grundgesetz. Band 1, 7. Aufl. 2018, Art. 3 Abs. 3 GG Rn. 423 ff. 
In diesem Lichte sind die speziellen Gleichheitsnormen des Staatsorganisationsrechts, insbesondere Art. 38 Abs. 1 S. 1 GG für die Legislative und Art. 33 Abs. 2 GG für die Exekutive und Judikative, auszulegen. Die Wahlrechtsgleichheit kann also in Fällen, in denen es um die faktische Benachteiligung beim Zugang zum Staat geht, material interpretiert werden. Dann sind politische Gleichstellungsmaßnahmen, wie z.B. Paritätsgesetze, an diesem Maßstab und nicht an der formalen Wahlrechtsgleichheit zu messen. Differenzierungen beim Maßstab der Wahlrechtsgleichheit nimmt das Bundesverfassungsgericht beispielsweise bei der Unterscheidung zwischen der Erfolgswertgleichheit (Fallkonstellation Verhältniswahlrecht) und der Erfolgschancengleichheit (Fallkonstellation Mehrheitswahlrecht) vor. ${ }^{37}$

Die vorgeschlagene Perspektive kann darüber hinaus an die Rechtsprechung des Bundesverfassungsgerichts anschließen. In der Entscheidung zum Ausschluss von Vollbetreuten hat das Bundesverfassungsgericht entschieden, dass Wahlrechtsausschlüsse nicht nur am Grundsatz der Gleichheit der Wahl, sondern auch am Diskriminierungsverbot von Menschen mit Behinderung gem. Art. 3 Abs. 3 S. 2 GG zu messen sind. ${ }^{38}$ Die Wahlrechtsgleichheit und der besondere Diskriminierungsschutz aus Art. 3 Abs. 3 GG seien nebeneinander anwendbar, weil sie unterschiedlichen Schutzzwecken dienen: Der Egalität der Staatsbürger:innen und der Nichtdiskriminierung von Menschen mit Behinderung. ${ }^{39}$ Beim Zugang von Frauen zu den Parlamenten geht es genau um die Überschneidung dieser Schutzzwecke, nämlich um die Beeinträchtigung der staatsbürgerlichen Egalität durch strukturelle Diskriminierung aufgrund des Geschlechts. Die Auslegung von Art. 38 Abs. 1 S. 1 GG im Lichte von Art. 3 Abs. 2 und 3 GG wäre daher eine konsequente Weiterentwicklung dieser Rechtsprechung.

Schließlich kann auch das Demokratieprinzip gem. Art. 20 Abs. 2 GG im Lichte materialer Gleichheit interpretiert werden. Aus einer individualrechtlichen Perspektive ${ }^{40}$ garantiert das Prinzip repräsentativer Demokratie nicht nur die formale Gleichheit der Staatsbürger:innen, sondern umfasst darüberhinaus eine gewährleistende Dimension: Es gewährleistet die

37 BVerfG, Urt. v. 10.04.1997 - 2 BvF 1/95, BVerfGE 95, 335 (352 ff., 369 f.), Überhangmandate. Kritisch zu einer materialen Perspektive im Wahlrecht Volk, DÖV 2021, 413.

38 BVerfG, Beschl. v. 29.01.2019 - 2 BvC 62/14.

39 Ebenda, Rn. $49 \mathrm{ff}$.

40 Zur Individualisierung der Demokratie in der Europarechtsprechung des BVerfG siehe Brade in diesem Band. 
grundsätzliche Möglichkeit der gleichen Teilhabe an den drei Staatsgewalten durch einen diskriminierungsfreien Zugang zum demokratischen Staat. ${ }^{41}$

\section{Paritätsgesetze: Geschlechtliche Repräsentation oder demokratische Gleichheit?}

Paritätsgesetze setzen beim Zugang zur Demokratie an: Sie verpflichten die Parteien dazu, eine gleiche Anzahl von Männern und Frauen für eine Wahl aufzustellen. ${ }^{42}$ Sie bilden damit ein Gegengewicht zur männlich geprägten politischen Kultur in den Parteien, die bisher männliche Karrieren in der Politik gefördert hat. Paritätsgesetze dienen daher auch dazu, gewachsene Privilegienstrukturen in den demokratischen Prozessen abzubauen. ${ }^{43}$

Verfassungsrechtlich verfügt der parlamentarische Gesetzgeber bei der Ausgestaltung des Wahlrechts auf der Grundlage des Art. 38 Abs. 3 GG über einen weiten Entscheidungsspielraum. Die Legislative muss aufgrund von Art. 3 Abs. 2 S. 2 GG die politische Gleichheit von Frauen im Staat fördern und auf die Beseitigung bestehender Nachteile hinwirken. Sie darf daher, muss aber nicht, Paritätsgesetze erlassen, weil sich diese - das zeigt der internationale Vergleich - als effektives Instrument zur Durchsetzung politischer Gleichheit erwiesen haben. ${ }^{44}$ Angesichts der stagnierenden und zum Teil auch erheblich sinkenden Frauenanteile in den Parlamenten sind Paritätsgesetze erforderlich und zum jetzigen Zeitpunkt verhältnismäßig. ${ }^{45}$

41 Röhner (Fn. 16), S. 291 ff.; dies., djbZ 22 (2019), 125.

$42 \mathrm{Zu}$ den Regelungsgehalten der deutschen Paritätsgesetze siehe Röhner, Discussion Paper des Harriet Taylor Mill-Instituts für Ökonomie und Geschlechterforschung, Nr. 40, 1/2020, 44 ff.; dies., Streit 2020, 57.

43 Die Auseinandersetzungen innerhalb der CDU um die geschlechtergerechte Besetzung der aussichtsreichen Listenplätze im Vorfeld der letzten Landtagswahlen in Brandenburg und Sachsen-Anhalt zeigen exemplarisch, dass sich Frauen durchaus um attraktive Listenplätze bewerben, sich aber ohne Quote oftmals nicht durchsetzen können, Sauerbier, Brandenburger CDU kippt Frauenliste, BZ online v. 16.06.2019; Nimz, Kerstin Rinke, SZ online v. 07.03.2021.

44 Eine Verpflichtung zum Erlass eines Paritätsgesetzes annehmend Laskowski, djbZ 2014, 93-103, dies., Streit 2015, 51-62.

45 Sie z.B. die Beiträge von Geppert, Fontana und Völzmann in djbZ 2019, Heft 3. Die demokratische Politik hätte aber auch andere Handlungsmöglichkeiten, um die Gleichheit der Geschlechter in den Parlamenten zu stärken. So ist beispielsweise 
Als antidiskriminierungsrechtliche Instrumente werden Paritätsgesetze bisher jedoch nur wenig diskutiert. Stattdessen wird primär die Forderung nach einer paritätischen Repräsentation bzw. der Spiegelbildlichkeit in den Parlamenten in den Vordergrund gestellt. Kritiker:innen lehnen daher Paritätsgesetze als Verstoß gegen das unitäre Repräsentationsverständnis ab. ${ }^{46}$ Nach dem unitären Repräsentationsverständnis repräsentieren die Abgeordneten das gesamte Volk, weshalb auch Frauen ausreichend mitrepräsentiert seien. Das freie Mandat verpflichte die Abgeordneten zudem nur auf ihr Gewissen und nicht auf die Repräsentation von spezifischen Geschlechterinteressen.

Während diese Argumentationslinie die landesverfassungsrechtliche Rechtsprechung zu Paritätsgesetzen dominiert (1.), betont das Bundesverfassungsgericht die Möglichkeit einer antidiskriminierungsrechtlichen Rechtfertigung von Paritätsgesetzen (2.).

\section{Landesverfassungsrechtliche Rechtsprechung}

Paritätsgesetze wurden bisher von den Landesverfassungsgerichten in Bayern, Thüringen und Brandenburg verhandelt. Alle Entscheidungen fielen negativ aus. Die Entscheidungsbegründungen sind davon geprägt, $\mathrm{Pa}$ ritätsgesetze als Instrument zur geschlechtlichen Repräsentation anstatt als demokratisches Antidiskriminierungsrecht zu verstehen. Es fällt auf, dass die Gerichte nicht in der Lage - oder nicht bereit - sind, die Geschlechterungleichheit als Problem demokratischer Gleichheit zu verhandeln und strukturelle Ungleichheit in staatlichen Institutionen zu adressieren. Stattdessen betonen sie, dass aufgrund des unitären Repräsentationsverständnisses und der formalen Gleichheit im Staat verfassungsrechtlich kein relevantes Gleichheitsproblem bestehe. ${ }^{47}$

ein reines Verhältniswahlrecht frauen- und minderheitenfreundlicher, m.w.N. Röhner (Fn. 16), S. 269 ff.

46 Zur Kritik des Repräsentationsbegriffs und warum dieser in der Debatte nicht weiterführend ist, ausführlich Röhner, Der Staat 59 (2020), 421 (425 ff.).

47 Ausführlich und kritisch zu den Entscheidungen aus Thüringen und Brandenburg Möllers, JZ 2021, 338; Völzmann, DVBl 2021, 496; Klafki, DÖV 2020, 856; siehe auch Edinger, DÖV 2021, 442. 
a) Die Entscheidungen im Überblick: Paritätsgesetze als geschlechtliche Repräsentation

Der Bayerische Verfassungsgerichtshof entschied im Jahr 2018 negativ über eine Popularklage, die das Fehlen einer paritätischen Ausgestaltung der Regelungen zum Wahlvorschlagsrecht der Parteien in Bayern zum Gegenstand hatte und das Ziel verfolgte, den bayerischen Gesetzgeber auf den Erlass eines Paritätsgesetzes zu verpflichten..$^{48}$ Die negative Entscheidung überzeugt im Ergebnis, weil die Legislative verfassungsrechtlich nicht auf den Erlass eines Paritätsgesetzes verpflichtet werden kann. ${ }^{49}$

In der Argumentation des Bayerische Verfassungsgerichtshofs dominieren jedoch die Auseinandersetzung mit Paritätsgesetzen als Instrument geschlechtlicher Repräsentation. So legt der Bayerische Verfassungsgerichtshof dar, dass eine "gewisse « Unterrepräsentation von Frauen verfassungsrechtlich nicht zu beanstanden sei, weil es aufgrund des Prinzips repräsentativer Demokratie und des freien Mandats kein Recht einer Bevölkerungsgruppe auf Repräsentation gäbe. ${ }^{50}$ Zwar erkennt das Gericht durchaus an, dass inzwischen ein materiales Diskriminierungsverständnis entwickelt wurde, eine angemessene Prüfung einer mittelbaren Diskriminierung, wie sie dem aktuellen Diskussions- und Rechtsprechungsstand entspräche, führt das Gericht jedoch nicht durch. Stattdessen zeigt sich ein deutlicher Widerwille des Gerichts, eine antidiskriminierungsrechtliche Perspektive im Wahlrecht zuzulassen und zu prüfen. ${ }^{51}$

Über die Verfassungsmäßigkeit eines Paritätsgesetzes entschied erstmals der Thüringer Verfassungsgerichtshof im Jahr 2020. Mit einem Votum von 6:3 erklärte das Gericht das thüringische Paritätsgesetz für verfassungswidrig. ${ }^{52}$ In der Begründung der Senatsmehrheit ist die Argumentationslinie einer unitären Repräsentation und formalen Wahlrechtsgleichheit vorherrschend während eine antidiskriminierungsrechtliche Perspektive - konträr zur bayerischen Entscheidung - vollständig fehlt. Die Senatsmehrheit nimmt zahlreiche Verfassungsverstöße durch das Thüringische Paritätsgesetz an, thematisiert jedoch nicht die Unterrepräsentation von Frauen im Landtag und in den kommunalen Vertretungen in Thüringen. Sie disku-

48 Bayerischer Verfassungsgerichtshof, Ent. v. 26.05.2018 - Vf. 15-VII-16.

49 Ebenda, Rn. 133.

50 Ebenda, Rn. $99 \mathrm{ff} ., 110 \mathrm{ff}$.

51 Ausführlich Röhner, (Fn. 16), S. 283 ff.

52 Thüringer Verfassungsgerichtshof, Urt. v. 13.05.2020 - VerfGH 2/2020; dazu Möllers, JZ 2021, 338 (340 ff.); Völzmann, DVBl 2021, 496 (498ff.); Klafki, DÖV 2020, 856. 
tiert dementsprechend weder die Ursachen für die Unterrepräsentation von Frauen noch den Sinn und Zweck des Paritätsgesetzes, einen diskriminierungsfreien Zugang zum Parlament zu gewährleisten und Gleichberechtigung herzustellen. In der Entscheidungsbegründung des Senats ist nicht zu erkennen, dass das Problem der politischen Ungleichheit von Frauen in Thüringen existiert. Die Senatsmehrheit macht damit die Ungleichheit von Frauen in den thüringischen Vertretungsorganen unsichtbar und definiert diese nicht als Verfassungsproblem. Eine ausführliche Abwägung zwischen den Beeinträchtigungen und Zielen des Gesetzes normalerweise das Herzstück einer verfassungsrechtlichen Entscheidung findet daher nicht statt.

Anders argumentieren die zwei ergangenen Sondervoten. ${ }^{53}$ Beide benennen die politische Ungleichheit von Frauen und sehen im Gleichstellungsauftrag in Art. 2 Abs. 2 S. 2 der Thüringischen Verfassung eine ausreichende verfassungsrechtliche Rechtfertigung für das thüringische Paritätsgesetz. Die Landesverfassungsrichter:innen Licht und Petermann betonen in ihrem Sondervotum die strukturellen Diskriminierungserfahrungen von Frauen in den politischen Prozessen und zeigen auf, dass aufgrund der Rechtsprechung des Bundesverfassungsgerichts nicht mehr ein formales, sondern ein materiales Gleichheitsverständnis für die Bewertung von Paritätsgesetzen maßgeblich sei. ${ }^{54}$

Das Verfassungsgericht des Landes Brandenburg erklärte im Jahr 2020 das brandenburgische Paritätsgesetz einstimmig für verfassungswidrig. ${ }^{55}$ Es war ebenfalls der Auffassung, dass ein Paritätsgesetz nicht mit dem Prinzip repräsentativer Demokratie und der formalen Wahlrechtsgleichheit zu vereinbaren sei. Es argumentiert, dass das Paritätsgesetz zu einem anderen Verständnis von repräsentativer Demokratie führe und ein solches nur vom Verfassungsgesetzgeber eingeführt werden könne. ${ }^{56}$ Die Durchsetzung der Gleichberechtigung sei ein demokratie- und wahlrechtsfremder Zweck. Der Gleichstellungsauftrag könne daher die Beeinträchtigungen durch das Paritätsgesetz nicht rechtfertigen. ${ }^{57}$ Das Verfassungsgericht des Landes Brandenburg bewertet also das Gleichstellungsgebot als we-

53 Thüringer Verfassungsgerichtshof, Urt. v. 13.05.2020 - VerfGH 2/2020, S. 46 ff.

54 Ebenda, S. $52 \mathrm{ff}$.

55 Verfassungsgericht des Landes Brandenburg, Urt. v. 23.10.2020 - VfgBbg 9/19, Parallelentscheidung VfgBbg 55/19. Dazu Möllers, JZ 2021, 338 (340 ff.); Völzmann, DVBl 2021, 496 (498 ff).

56 Verfassungsgericht des Landes Brandenburg, Urt. v. 23.10.2020 - VfgBbg 9/19, Rn. 86.

57 Ebenda, Rn. $115 \mathrm{ff}$. 
niger schwergewichtig als die Wahlrechtsgrundsätze und beschreibt die faktischen Diskriminierungserfahrungen von Frauen in den politischen Prozessen als kein Problem demokratischer Gleichheit. Erneut fehlt es an einer ausführlichen Abwägung. Die Prüfung wird bereits vorher beendet, u.a. weil das Gericht ungewöhnlicher Weise fordert, dass der Wortlaut der Landesverfassung die Einführung einer Quotierung im Wahlrecht ausdrücklich erlauben müsse. Erneut wird vom Gericht eine antidiskriminierungsrechtliche Auslegung im Wahlrecht nicht zugelassen.

\section{b) Kritik: Paritätsgesetze als antidiskriminierungsrechtliche Instrumente}

In den negativen Entscheidungen manifestiert sich die bisher maskulin strukturierte epistemische Ordnung des Verfassungsrechts. Nicht nur in den Parlamenten, sondern auch in der Verfassungsgerichtsrechtsprechung und Verfassungswissenschaft sind Frauen unterrepräsentiert, weshalb die Geschlechterungleichheit in der Demokratie bis zur aktuellen Paritätsdebatte $^{58}$ in der verfassungsrechtlichen Literatur dethematisiert wurde. ${ }^{59}$ Dementsprechend wird die Geschlechterungleichheit in den Parlamenten von der "herrschenden Meinung“ bisher nicht als Problem von Demokratie und demokratischer Gleichheit verstanden. Diese Leerstelle konnten u.a. rechtsextremen Parteien für sich nutzen, um vor den Landesverfassungsgerichten gegen Paritätsgesetze vorzugehen. Die Erfolge vor den Landesverfassungsgerichten zeigen, dass die Geschlechterungleichheit in der verfassungsrechtlichen Literatur reale Konsequenzen für die Realisierung von politischer Gleichheit hat.

Aus verfassungsrechtlicher Sicht überzeugt der Fokus der Landesverfassungsgerichte auf geschlechtliche Repräsentation angesichts des antidiskriminierungsrechtlichen Gleichstellungszwecks von Paritätsgesetzen nicht. Indem Paritätsgesetze die Parteien darauf verpflichten, eine gleiche Anzahl von Männern und Frauen nach dem Reißverschlussprinzip aufzustellen, gewährleisten sie, dass Frauen die gleiche Chance haben, ins Parlament gewählt zu werden wie männliche Kandidaten. Sie sichern also, dass Frauen keine Nachteile aufgrund ihres Geschlechts beim Zugang zum Parlament

58 Siehe allgemein Eckertz-Höfer/Schuler-Harms (Hrsg.), Gleichberechtigung und Demokratie - Gleichberechtigung in der Demokratie: (Rechts-)Wissenschaftliche Annäherungen, 2019; zur aktuellen Paritätsdebatte die Nachweise in Fn. 42, Fn. 45, Fn. 52 sowie Meyer, NVwZ 2019, 1245; Morlok/Hobusch, DÖV 2019, 14.

59 Zur Wissenschaft Sacksofsky/Stix, KJ (2018), 464; zum verfassungsrechtlichen Diskurs m.w.N. Röhner (Fn. 16), S. 267 ff. 
haben. Sie führen jedoch zu keiner geschlechtlichen Repräsentation, weil sie keinen Mechanismus einführen, nachdem Frauen ihre Repräsentantinnen und Männer ihre Repräsentanten wählen. Auch hätten die bisher erlassenen Paritätsgesetze zu keiner Parität in den Parlamenten im Sinne von Ergebnisgleichheit geführt, da keine Quotierung für die Direktmandate vorgesehen war. ${ }^{60}$

Darüber hinaus verkennen die Landesverfassungsgerichte, dass Paritätsgesetze mit einem unitären Repräsentationsverständnis vereinbar sind, weil sie nur sichern, dass auch Frauen Teil der privilegierten Gruppe der Abgeordneten werden, die das gesamte Volk repräsentiert und im Rahmen des freien Mandats die politischen Entscheidungen trifft. Mit Paritätsgesetzen wird also weder die Fiktion einer unitären Repräsentation aufgegeben noch werden die gewählten Abgeordneten an die Vertretung geschlechtlicher Interessen gebunden.

Verfassungsrechtlich kann eine antidiskriminierungsrechtliche Perspektive im Wahlrecht - und allgemeiner im staatlichen Bereich - eröffnet werden, wenn die vorherrschende formale Perspektive historisiert und kontextualisiert wird. In den skizzierten Entscheidungen wird bisher nämlich nicht reflektiert, dass das unitäre Repräsentationsverständnis und die formale Wahlrechtsgleichheit in der spezifischen Konstellation der bürgerlichen Revolutionen entstanden sind und daher in den Konflikten zwischen Bürgertum und Monarchie sowie zwischen repräsentativer und direkter Demokratie gründen. Sie reagieren auf die soziale Differenzierung von Rechten im Ancien Régime und auf den Ausschluss aus dem Wahlrecht aufgrund von sozialen Zugehörigkeiten. Als verfassungsrechtliche Maßstäbe klären sie aber historisch betrachtet nicht die Anforderungen, die sich in einer demokratischen, aber ungleichen Gesellschaft für die Gleichheit im Staat ergeben. Eine Erweiterung um eine materiale Perspektive für die „neue“ Fallgruppen der faktischen Benachteiligung beim Zugang zu Staatsämtern ist daher möglich und aufgrund des materialen Gleichheitsverständnisses des Bundesverfassungsgerichts auch angezeigt.

60 Zur fehlenden Quotierung der Direktmandate und der Unterscheidung zwischen Chancen- und Ergebnisgleichheit Röhner (Fn. 42 - Discussionpaper), S. $23 \mathrm{f}$, $30 \mathrm{ff}$.; dies., Streit 2020, 57, (62 f., 65 f.). 


\section{Beschluss des Bundesverfassungsgerichts}

Ein materiales Gleichheitsverständnis für den Zugang zu Staatsämtern eröffnet das Bundesverfassungsgericht in seinem Beschluss vom 15. Dezember 2020 - 2 BvC 46/19. In dem Wahlprüfungsbeschwerdeverfahren ging es nicht darum, ob Paritätsregelungen verfassungsrechtlich zulässig sind, sondern ähnlich wie im bayerischen Fall darum, ob das Fehlen einer paritätischen Ausgestaltung des Wahlvorschlagrechts bei der Bundestagswahl 2017 einen schwerwiegenden Wahlfehler darstellt, weil nur 30 Prozent der Sitze an Frauen gingen. Überzeugenderweise verneint das Bundesverfassungsgericht diese Frage. Es gibt zugleich Hinweise zur Rechtfertigung von Paritätsgesetzen: Das Bundesverfassungsgericht legt ausführlich dar, dass es die Rechtfertigung über das Erfordernis einer spiegelbildlichen Repräsentation für nicht überzeugend hält ${ }^{61}$ aber durchaus offen für eine antidiskriminierungsrechtliche Begründung ist. ${ }^{62}$

Anders als die Landesverfassungsgerichte sieht das Bundesverfassungsgericht im Gleichstellungsauftrag ein mit den Wahlrechtsgrundsätzen und der Parteifreiheit gleichrangiges Verfassungsgut. ${ }^{63}$ Es betont daher konträr zu den bisherigen Entscheidungen, dass es die Sache des Gesetzgebers sei, die verschiedenen Verfassungsgüter im Rahmen des Wahlrechts zu einem angemessenen Ausgleich zu bringen. ${ }^{64}$ Es macht deutlich, dass es nach seiner Ansicht für die Verfassungsmäßigkeit entscheidend auf die Abwägung und das Ziel ankommen wird, strukturelle Nachteile von Frauen in den Nominierungsverfahren der politischen Parteien auszugleichen. ${ }^{65}$

Für zukünftige Paritätsgesetze - und andere antidiskriminierungsrechtliche Maßnahmen, wie etwa die Berliner Diversitätsquote - dürften daher die faktischen Nachteile in der sozialen Wirklichkeit der Ausgangspunkt und die zentrale Rechtfertigung sein. Auch die Bewertung, ob eine strukturelle Benachteiligung vorliegt, dürfte nach der Entscheidung des Bundesverfassungsgerichts im Einschätzungsspielraum der demokratischen Politik liegen. Im Kern zeigt der Beschluss des Bundesverfassungsgerichts, dass sich die Legislative grundsätzlich für die Einführung eines Paritätsgesetzes zur Durchsetzung der demokratischen Gleichheit von Frauen entscheiden

61 BVerfG, Beschl. v. 15.12.2020 - 2 BvC 46/19, Rn. 64 ff. Dazu Penz, DÖV 2021, 422.

62 Ebenda, Rn. $84 \mathrm{ff}$.

63 Ebenda, Rn. $97 \mathrm{ff}$.

64 Ebenda, Rn. 109 ff., 113.

65 Valentiner, How to... Paritätsgesetz, VerfBlog v. 4.2.2021, DOI: $10.17176 / 20210204-120114-0$. 
darf, es sich dabei also um eine politische Entscheidung handelt, die verfassungsrechtlich nicht von vornherein ausgeschlossen ist.

\section{Ausblick}

Politische Maßnahmen, die vielfältigen Subjektivitäten den Zugang zu staatlichen Ämtern gewährleisten sollen, können in einem demokratischen Antidiskriminierungsrecht verankert werden. Angesichts der anhaltenden Unterrepräsentation dominierter Gruppen im Staat allgemein und insbesondere in hohen staatlichen Ämtern, dürfte eine Debatte um effektive Gleichstellungsmaßnahmen, insbesondere um Quotenregelungen für Frauen und andere dominierte Gruppen, intensiver zu führen sein. Die Paritätsgesetze aus Thüringen und Brandenburg, die Berliner Diversitätsquote sowie das aktuell im Gesetzgebungsverfahren befindliche Führungspositionengesetz II $^{66}$ für die Wirtschaft sind dafür ein Anfang. Die Widerstände gegen solche effektiven Maßnahmen sind jedoch groß - dies zeigt das Scheitern der Diversitätsquote in Berlin und der Paritätsgesetze in Thüringen und Brandenburg. Denn: Es geht nicht nur um die Anerkennung als Gleiche, sondern auch um die Umverteilung von wirtschaftlicher und politischer Macht.

Verfassungsrechtlich können antidiskriminierungsrechtliche Instrumente für den Zugang zu staatlichen Ämtern durch ein materiales Gleichheitsverständnis begründet werden. Ein solches versteht strukturelle Ungleichheit im Staat als demokratisches Gleichheitsproblem. Es verdeutlicht, dass es in einer pluralistischen Demokratie eines gleichberechtigten Zugangs bedarf, um für alle Staatsbürger:innen, unabhängig von ihrer gesellschaftlichen Positionierung, eine gleichberechtigte Teilhabe an den drei Staatsgewalten zu gewährleisten - und in diesem Sinne gerechte Staatlichkeit zu erreichen.

66 BT-Drucksache 19/26689. 
